\title{
Modules which are self-p-injective relative to projection invariant submodules
}

\author{
Yeliz Kara and Adnan Tercan
}

\begin{abstract}
In this article, we focus on modules $M$ such that every homomorphism from a projection invariant submodule of $M$ to $M$ can be lifted to $M$. Although such modules share some of the properties of $P I$-extending (i.e., every projection invariant submodule is essential in a direct summand) modules, it is shown that they form a substantially bigger class of modules.
\end{abstract}

\section{Introduction}

Throughout this paper, let $R$ be a ring with identity and let all modules be unitary right $R$-modules. Let $M$ be a module. The injective hull of $M$ is denoted by $E(M)$. A submodule $K$ of $M$ is projection invariant (denoted by $K \unlhd_{p} M$ ) provided $K$ is invariant under every idempotent endomorphism of $M$ (see [3], [5]). Note that the set of projection invariant submodules of a module $M$ forms a sublattice of the lattice of all submodules of $M$.

A module $M$ is called an extending module, or a $C S$-module, if every submodule of $M$ is essential in a direct summand, or, equivalently, if every closed submodule of $M$ is a direct summand. This condition has proven to be an important common generalization of the injective and semisimple module notions (see, [4], [11]). In [3], the extending condition relative to various sets of submodules have been investigated. Recall that a module $M$ is called PI-extending

Key Words: injective module, projection invariant submodule, PI-extending module, extending module

2010 Mathematics Subject Classification: Primary 16D10; Secondary: 16D40, 16D50

Received: 21.02.2016

Accepted: 20.04.2016 
if every projection invariant submodule is essential in a direct summand of $M$. In the papers [14] and [16], the authors studied the following property, for a module $M$ :

$\left(P_{n}\right)$ : For every submodule $K$ of $M$ such that $K$ can be written as a finite direct sum $K_{1} \oplus K_{2} \oplus \ldots \oplus K_{n}$ of complements $K_{1}, K_{2}, \ldots, K_{n}$ of $M$, every homomorphism $\alpha: K \rightarrow M$ can be lifted to a homomorphism $\beta: M \rightarrow M$. Following an idea from [16], we are concerned with the study of self-p-injective modules, i.e., modules $M$ that satisfy the condition that every homomorphism from a projection invariant submodule of $M$ to $M$ can be lifted to $M$. Observe that the aforementioned property is equivalent to that of every homomorphism from a finite direct sum of projection invariant submodules of $M$ to $M$ lifts to $M$. Extending and $P I$-extending modules are examples of self-p-injective modules. Our investigation focuses on the behavior of self-p-injective modules with respect to direct sums and direct summands. To this end, we provide algebraic geometrical examples which show that being self-p-injective is not inherited by direct summands. In contrast, we prove that any direct sum of self-p-injective modules enjoys with the property. Moreover we obtain useful characterizations and direct sum property on relatively p-injective modules. Finally, we give examples which show that there is no implication between self-p-injective and tight concepts. Recall that a module $M$ is said to be right tight (resp.,right $M$-tight) if every finitely generated (resp., cyclic) submodule of $E(M)$ can be embedded in $M$ (see [1], [6]).

Recall the following conditions for a module $M$.

$\left(C_{2}\right)$ : every submodule of $M$ can be embedded in a direct summand of $M$.

$\left(C_{3}\right)$ : for all direct summands $K$ and $L$ of $M$ with $K \cap L=0$, the submodule $K \oplus L$ is also a direct summand of $M$.

Observe that $C_{2}$ implies $C_{3}$ by [11, Proposition 2.2]. Recall further that, a ring is called Abelian if every idempotent is central. Other terminology and notation can be found in [2], [4], [10], and [11].

\section{Direct Summands and Direct Sums}

In this section, we concern ourselves with direct summands and direct sums of self-p-injective modules. We provide examples which show that, in general, direct summands of a self-p-injective module need not to be self-p-injective. Amongst some affirmative answers for the former closure property we also prove that any direct sum of self-p-injective modules is again self-p-injective.

Lemma 2.1. Let $M$ be an indecomposable module. Then the following statements are equivalent.

(i) $M$ is quasi-injective. 
(ii) $M$ is extending.

(iii) $M$ is PI-extending.

(iv) $M$ is self-p-injective.

Proof. $(i) \Rightarrow$ (ii) Obvious.

(ii) $\Rightarrow$ (iii) Clear from [3, Proposition 3.7].

(iii) $\Rightarrow($ iv) Let $X$ be a projection invariant submodule of $M$ and $\varphi: X \rightarrow$ $M$ be a homomorphism. Then there exists a direct summand $D$ of $M$ such that $X$ is essential in $D$ where $M=D \oplus D^{\prime}$. Let $\pi$ be projection map on $X$ in $D$. Then define $\alpha: M \rightarrow M$ such that $\alpha=\varphi \pi$. It can be easily seen that $\alpha$ lifts $\varphi$. Hence $M$ is self-p-injective.

$(i v) \Rightarrow(i)$ Since $M$ is an indecomposable module, every submodule of $M$ is projection invariant. Then it is clear that self-p-injectivity implies quasiinjectivity.

Observe that every quasi-injective module is self-p-injective. However there are self-p-injective modules which are not quasi-injective. For example let $M_{\mathbb{Z}}=(\mathbb{Z} / \mathbb{Z} p) \oplus \mathbb{Q}$ where $p$ is any prime integer. Then $M_{\mathbb{Z}}$ is not quasi-injective but it is self-p-injective by Theorem 2.8. Note that every $P I$-extending module is self-p-injective. But the converse of this result is not true, in general. For instance, let $M$ be the Specker group, $M_{\mathbb{Z}}=\prod_{i=1}^{\infty} A_{i}$ with $A_{i}=\mathbb{Z}$ for any positive integer $i$. Then it can be checked that $M_{\mathbb{Z}}$ is not $P I$-extending by [5], but it is self-p-injective by Theorem 2.14 .

The next example shows that direct summands of self-p-injective modules need not to be self-p-injective, in general.

Example 2.2. (See, [3, Example 5.5] or [17, Example 4]) Let $\mathbb{R}$ be the real field and $n$ be any odd integer with $n \geq 3$. Let $S$ be the polynomial ring $\mathbb{R}\left[x_{1}, \ldots, x_{n}\right]$ over $\mathbb{R}$ in indeterminates $x_{1}, \ldots, x_{n}$. Let $R$ be the ring $S / S s$, where $s=x_{1}^{2}+\ldots+x_{n}^{2}-1$. Then the free $R$ - module, the countable direct sum $M=R^{(\mathbb{N})}$ of copies of $R$ is self-p-injective which contains a direct summand $K_{R}$ which is not self-p-injective.

Surprisingly, we may provide more examples in the next result which is based on certain hypersurfaces in projective spaces, $\mathbb{P}_{\mathbb{C}}^{\text {n+1 }}$ over complex numbers.

Theorem 2.3. Let $X$ be the hypersurface in $\mathbb{P}_{\mathbb{C}}^{n+1}, n \geq 2$, defined by the equation $x_{0}^{m}+x_{1}^{m}+\ldots+x_{n+1}^{m}=0$. Let $R=\mathbb{C}\left[x_{1}, \ldots, x_{n+1}\right] /\left(\sum_{i=1}^{n+1} x_{i}^{m}+1\right)$ be the coordinate ring of $X$. There exist self-p-injective $R$-modules but contain direct summands which are not self-p-injective for $m \geq n+2$. 
Proof. By [12], there are indecomposable projective $R$-modules of rank $n$ over $R$. It follows that $F_{R}=K \oplus W$ where $F_{R}$ is a free module, $K$ is indecomposable and projective $R$-module of rank $n$. From [3, Corollary 4.11], $F_{R}$ is $P I$-extending and hence it is self-p-injective. Now $K_{R}$ is not uniform. Thus $K_{R}$ is not $P I$-extending so it is not self-p-injective by Lemma 2.1 .

However, we deal with some special cases when the self-p-injectivity is inherited by direct summands in the following results.

Proposition 2.4. Let $M=M_{1} \oplus M_{2}$ where $M_{1}$ and $M_{2}$ are projection invariant submodules of $M$. If $M$ is self-p-injective then $M_{1}, M_{2}$ are also selfp-injective.

Proof. Let $N_{1}$ be a projection invariant submodule of $M_{1}$ and $\varphi: N_{1} \rightarrow M_{1}$ be a homomorphism. Since $N_{1}$ is projection invariant submodule of $M_{1}$ and $M_{1}$ is projection invariant submodule of $M$, then $N_{1}$ is projection invariant submodule of $M$. Observe that $\iota \varphi: N_{1} \rightarrow M$ where $\iota$ is inclusion map. Then there exists $\theta: M \rightarrow M$ such that $\theta$ lifts to $\iota \varphi$. Define $\gamma: M_{1} \rightarrow M_{1}$ by $\gamma\left(m_{1}\right)=\theta\left(m_{1}\right)$. It is clear that $\varphi$ can be extended to $\gamma$. Then $M_{1}$ is self-pinjective. Similarly, it can be shown that $M_{2}$ is also self-p-injective.

Corollary 2.5. Let $M=M_{1} \oplus M_{2}$ for submodules $M_{1}$ and $M_{2}$ of $M$ with $S=$ $\operatorname{End}\left(M_{R}\right)$ an Abelian ring. If $M$ is self-p-injective then any direct summand of $M$ is also self-p-injective.

Proof. Let $\pi: M \rightarrow M_{2}$ be projection map with $\operatorname{ker}(\pi)=M_{1}$. Let $e=e^{2} \in S$. Since $S$ is Abelian, $e(\operatorname{ker}(\pi)) \subseteq \operatorname{ker}(\pi)$. Hence $M_{1}$ is projection invariant in $M$. Now, apply Proposition 2.4 which yields the corollary.

Proposition 2.6. Let $M=M_{1} \oplus M_{2}$ where $M_{1}, M_{2} \leq M$ such that $M_{2}$ is a projection invariant submodule of $M$. If $M$ is self-p-injective then $M_{1}$ is self-p-injective.

Proof. Let $N$ be projection invariant submodule of $M_{1}$ and $\varphi: N \rightarrow M_{1}$ be a homomorphism. Then $N \oplus M_{2}$ is projection invariant in $M$ by [3, Lemma 4.13]. Now consider $\theta=\iota \varphi \pi_{1}$ where $\pi_{1}: N \oplus M_{2} \rightarrow N$ is projection and $\iota: M_{1} \rightarrow M$ is inclusion. Thus there exists $\gamma: M \rightarrow M$ such that $\gamma$ lifts to $\theta$. Hence

$$
\gamma\left(n+m_{2}\right)=\theta\left(n+m_{2}\right)=\iota \varphi \pi_{1}\left(n+m_{1}\right)=\varphi(n) .
$$

Define $\tau: M_{1} \rightarrow M_{1}$ by $\tau=\pi \beta$ where $\beta$ is a restriction of $\theta$ to $M_{1}$ and $\pi: M \rightarrow M_{1}$. Then let $n \in N . \tau(n)=\pi \beta(n)=\pi \theta(n)=\varphi(n)$. Thus $\varphi$ can be extended to $\tau$ so $M_{1}$ is self-p-injective. 
Proposition 2.7. Let $M=M_{1} \oplus M_{2}$ for submodules $M_{1}, M_{2}$ of $M$. If $M_{1}$ is self-p-injective then $\varphi: N \rightarrow M$ can be lifted to $\theta: M \rightarrow M$ for all projection invariant submodule $N$ of $M_{1}$.

Proof. Let $N$ be projection invariant submodule of $M_{1}$ and $\varphi: N \rightarrow M$ be a homomorphism. Then $\pi_{1} \varphi \in \operatorname{Hom}\left(N, M_{1}\right)$ where $\pi_{1}: M \rightarrow M_{1}$. Then there exists $\theta: M_{1} \rightarrow M_{1}$ such that $\pi_{1} \varphi$ can be lifted to $\theta$. Define $\gamma: M \rightarrow M$ by $\gamma=\iota \theta \pi_{1}$. It is easy to check that $\gamma$ lifts to $\varphi$.

Theorem 2.8. Any direct sum of self-p-injective modules is self-p-injective.

Proof. Let $M_{\lambda}(\lambda \in \Lambda)$ be a nonempty collection of self-p-injective modules. Let $M=\underset{\lambda \in \Lambda}{\oplus} M_{\lambda}$ and $\varphi: N \longrightarrow M$ be homomorphism where $N$ is a projection invariant submodule of $M$. Let $\Lambda^{\prime}$ be a nonempty subset of $\Lambda$. Consider the set

$$
\mathcal{H}=\left\{\left(\Lambda^{\prime}, K^{\prime}, \alpha^{\prime}, \theta^{\prime}\right) \mid \Lambda^{\prime} \subseteq \Lambda, K^{\prime} \unlhd_{p} M^{\prime}=\underset{\lambda \in \Lambda^{\prime}}{\oplus} M_{\lambda} \text { and } \alpha^{\prime}: K^{\prime} \rightarrow M^{\prime}\right.
$$
homomorphism with $\theta^{\prime}: M^{\prime} \rightarrow M^{\prime}$ such that $\theta^{\prime}$ lifts to $\left.\alpha^{\prime}\right\}$

which becomes a partially ordered set by the componentwise order $\leq$ defined by

$$
\begin{gathered}
\left(\Lambda_{1}, K_{1}, \alpha_{1}, \theta_{1}\right) \leq\left(\Lambda_{2}, K_{2} \alpha_{2}, \theta_{2}\right) \Leftrightarrow \Lambda_{1} \subseteq \Lambda_{2}, K_{1} \subseteq K_{2}, \\
\left.\alpha_{2}\right|_{K_{1}}=\alpha_{1} \text { and } \theta_{1}=\pi \theta_{2} \iota
\end{gathered}
$$

where $\pi$ is canonical projection from $\underset{\lambda \in \Lambda_{2}}{\oplus} M_{\lambda}$ to $\underset{\lambda \in \Lambda_{1}}{\oplus} M_{\lambda}$ and $\iota$ is natural inclusion from $\underset{\lambda \in \Lambda_{1}}{\oplus} M_{\lambda}$ to $\underset{\lambda \in \Lambda_{2}}{\oplus} M_{\lambda}$.

Since $M_{\lambda}$ is self-p-injective for all $\lambda \in \Lambda$, the identity map $\iota$ can extend to $\theta: M_{\lambda} \rightarrow M_{\lambda}$. Hence $\left(\{\lambda\}, M_{\lambda}, \iota, \theta\right) \in \mathcal{H}$ so $\mathcal{H} \neq \varnothing$. Applying Zorn's Lemma, we can find a maximal element $\left(\Lambda_{1}, K_{1}, \alpha_{1}, \theta_{1}\right)$ in $\mathcal{H}$.

We claim that $\Lambda=\Lambda_{1}$. Suppose not, then there exists $\mu \in \Lambda, \mu \notin \Lambda_{1}$. Let $\Lambda_{2}=\Lambda_{1} \cup\{\mu\}$ and $M^{\prime \prime}=\underset{\lambda \in \Lambda_{2}}{\oplus} M_{\lambda}=\underset{\lambda \in \Lambda_{1}}{\oplus} M_{\lambda} \oplus M_{\mu}=M^{\prime} \oplus M_{\mu}$. Since $M_{\mu}$ is self-p-injective, then for any projection invariant submodule $N_{\mu}$ of $M_{\mu}$ and $\alpha_{\mu}: K_{\mu} \rightarrow M_{\mu}$ homomorphism, there exists $\theta_{\mu}: M_{\mu} \rightarrow M_{\mu}$ such that $\theta_{\mu}$ extends to $\varphi_{\mu}$. Observe that $K_{1} \oplus K_{\mu}$ is projection invariant in $M^{\prime \prime}$. Consider the homomorphism $\gamma: K_{1} \oplus K_{\mu} \rightarrow M^{\prime \prime}$ such that $\gamma\left(k_{1}+k_{\mu}\right)=\alpha_{1}\left(k_{1}\right)+\alpha_{\mu}\left(k_{\mu}\right)$. It is clear that $\gamma$ extends to $\alpha_{1}$. Define $\theta: M^{\prime \prime} \rightarrow M^{\prime \prime}$ by $\theta=\theta_{1} \pi_{1}+$ $\theta_{\mu} \pi_{2}$ where $\pi_{1}: M^{\prime \prime} \rightarrow M^{\prime}$ and $\pi_{2}: M^{\prime \prime} \rightarrow M_{\mu}$. Let $m^{\prime} \in M^{\prime}$. Then $\pi \theta \iota\left(m^{\prime}\right)=\pi\left(\theta_{1} \pi_{1}\left(m^{\prime}\right)+\theta_{\mu} \pi_{2}\left(m^{\prime}\right)\right)=\pi \theta_{1}\left(m^{\prime}\right)=\theta_{1}\left(m^{\prime}\right)$. Hence $\theta_{1}=\pi \theta \iota$. Now $\left(\Lambda_{2}, K_{1} \oplus K_{\mu}, \gamma, \theta\right) \in \mathcal{H}$. Note that $\left(\Lambda_{1}, K_{1}, \alpha_{1}, \theta_{1}\right) \leq\left(\Lambda_{2}, K_{1} \oplus K_{\mu}, \gamma, \theta\right)$ 
which contradicts the maximalitiy of $\left(\Lambda_{1}, K_{1}, \alpha_{1}, \theta_{1}\right)$ in $\mathcal{H}$. Therefore $\Lambda=\Lambda_{1}$, so $M=\underset{\lambda \in \Lambda}{\oplus} M_{\lambda}$ is self-p-injective.

Corollary 2.9. Any direct sum of modules which are PI-extending (resp., quasi-injective, extending or uniform) is self-p-injective.

Proof. Immediate by Theorem 2.8 .

Corollary 2.10. Let $M$ be a right $R$-module and $M=U \oplus V$ where $U$ and $V$ are uniform submodules of $M$. Then every direct summand of $M$ is self-pinjective.

Proof. Let $0 \neq K$ be a direct summand of $M$. If $K=M$ then $K$ is selfp-injective from Corollary 2.9. If $K \neq M$ then $K$ is uniform. Hence $K$ is self-p-injective.

Theorem 2.11. Let $M$ be a $\mathbb{Z}$-module such that $M$ is a direct sum of uniform modules. Then every direct summand of $M$ is self-p-injective.

Proof. Let $N$ be a direct summand of $M$. Then $N$ is also a direct sum of uniform modules by [15, Theorem 5.5]. Now Corollary 2.9 yields that $N$ is also self-p-injective.

One might expect that an essential extension of a self-p-injective module is self-p-injective. However, the next example eliminates this situation.

Example 2.12. Let $R$ be a principial ideal domain. If $R$ is not a complete discrete valuation ring then there exists an indecomposable torsion-free $R$-module $M$ of rank 2 by [8, Theorem 19]. Hence there exist uniform $U_{1}, U_{2}$ submodules of $M$ such that $U_{1} \oplus U_{2}$ is essential in $M$. Then $U_{1} \oplus U_{2}$ is self-p-injective by Corollary 2.9. However $M$ is not self-p-injective by Lemma 2.1.

Lemma 2.13. Let $X=\prod_{i \in I} X_{i}$ be a direct product of modules of $X_{i}$ for each $i \in I$. If $N$ is a projection invariant submodule of $X$ then $N=\prod_{i \in I}\left(N \cap X_{i}\right)$.

Proof. It is straightforward to check.

Theorem 2.14. Let $X=\prod_{i \in I} X_{i}$. Then $X$ is self-p-injective if and only if $X_{i}$ is self-p-injective for all $i \in I$.

Proof. Let $N_{i}$ be projection invariant in $X_{i}$ and $\varphi: N_{i} \rightarrow X_{i}$ be a homomorphism. Then $T=\prod_{i \in I} N_{i}$ is projection invariant in $X$. Let $\alpha_{i}: T \rightarrow N_{i}$ be a projection map. Consider $\iota \varphi \alpha_{i}: T \rightarrow X$ where $\iota$ is inclusion. Then there 
exists $\theta: X \rightarrow X$ such that $\theta$ lifts $\iota \varphi \alpha_{i}$. Define $\gamma: X_{i} \rightarrow X_{i}$ by $\gamma=\pi_{i} \theta \iota$ where $\pi_{i}$ is projection map from $X$ to $X_{i}$. It is clear that $\gamma$ lifts to $\varphi$, so $X_{i}$ is self-p-injective.

Conversely, let $N$ be a projection invariant submodule of $X$ and $\varphi: N \rightarrow$ $X$ be a homomorphism. Then $N \cap X_{i}$ is projection invariant submodule of $X_{i}$. Let $\theta$ be the restriction of $\varphi$ to $N \cap X_{i}$. Then $\pi_{i} \theta: N \cap X_{i} \rightarrow X_{i}$ for all $i \in I$. Hence there exists $\gamma: X_{i} \rightarrow X_{i}$ such that $\gamma$ lifts to $\pi_{i} \theta$ for all $i \in I$. Define $\alpha: X \rightarrow X$ by $\alpha=\iota \gamma \pi_{i}$. Let $n \in N$. By Lemma 2.13, $n=$ $\left(\pi_{i}(n)\right)_{i \in I}$. Thus $\alpha(n)=\alpha\left(\left(\pi_{i}(n)\right)_{i \in I}\right)=\iota \gamma \pi_{i}\left(\left(\pi_{i}(n)\right)_{i \in I}\right)=\iota \gamma\left(\left(\pi_{i}(n)\right)_{i \in I}\right)=$ $\iota \pi_{i} \theta\left(\left(\pi_{i}(n)\right)_{i \in I}\right)=\theta\left(\left(\pi_{i}(n)\right)_{i \in I}\right)=\varphi\left(\left(\pi_{i}(n)\right)_{i \in I}\right)=\varphi(n)$. Hence $\alpha$ lifts to $\varphi$ so $X$ is self-p-injective.

The conditions $C_{2}$ and $C_{3}$ can be characterized by the lifting homomorphisms from certain submodules to the module itself, as was shown in [16]. We obtain relations between the class of modules which is self-p-injective and the class of modules which has $C_{3}$ condition. From [16, Lemma 1], we have the following implication for a module $M$.

$$
C_{3} \Rightarrow \text { self-p-injective }
$$

Note that this implication is not reversible. For example, let $M_{\mathbb{Z}}=\oplus_{i=1}^{\infty} \mathbb{Z}$. Then $M_{\mathbb{Z}}$ is self-p-injective by Theorem 2.8 , but $M_{\mathbb{Z}}$ does not satisfy $C_{3}$ by [16, Example 9].

The next few results, which generalize [9, Theorem 2.12$]$, concern the endomorphism ring of self-p-injective $\pi$-duo modules. We call a module $M$ is $\pi$-duo if every submodule is projection invariant in $M$. We will use $S$ and $J(S)$ to denote the endomorphism ring of a module $M$ and the Jacobson radical of $S$, respectively. Further $\Delta$ will stand for the ideal $\{\alpha \in S \mid \operatorname{ker}(\alpha)$ is essential in $M\}$.

Theorem 2.15. Let $M_{R}$ be a self-p-injective module and $S$ an Abelian ring. Then $S / \Delta$ is a (von Neumann) regular ring and $\Delta=J(S)$.

Proof. Let $f \in S$ and $K=\operatorname{ker} f$. Since $\operatorname{ker}(f) \cap \operatorname{ker}(1-f)=0$, there exists an isomorphism $\alpha: K \rightarrow(1-f) K$. Consider the inverse map of $\alpha$. Since $M$ is $\pi$-duo module, $(1-f) K$ is projection invariant in $M$. By hypothesis, there exists $g \in S$ such that $g$ lifts inverse map of $\alpha$. Then $g(1-f)(k)=k$ for all $k \in K$. Let $B$ be a complement of $\operatorname{ker}(f)$ in $M$. Note that $f$ restricts to an isomorphism of $B$ onto $f(B)$, since $B \cap \operatorname{ker}(f)=0$. Observe that $f(B)$ is also projection invariant submodule of $M$ as $S$ is Abelian. By self-pinjectivity of $M$, extend the inverse isomorphism $f(B) \rightarrow B$ to some $\gamma \in S$. Now, $\gamma(f(b))=b$ for all $b \in B$ and hence $(f \gamma f-f)(B)=0$. Moreover, $B \oplus \operatorname{ker}(f) \leq \operatorname{ker}(f \gamma f-f) \leq M$ which gives that $(f \gamma f-f) \in \Delta$. Hence $S / \Delta$ is a (von Neumann) regular ring. It is well known that, regular rings have zero radical, hence $J(S / \Delta)=0$. Since $J(S) / \Delta \subseteq J(S / \Delta)$, then $J(S)=\Delta$. 
Corollary 2.16. Let $M$ be a nonsingular $\pi$-duo right $R$-module. If $M$ is a self-p-injective module, then $S$ is a regular ring.

Proof. Let $g \in \Delta$ and $N=\operatorname{ker}(g)$. Then for any $x \in M$, build up the following set

$$
L=\{r \in R \mid x r \in N\}
$$

Then clearly $L$ is a right ideal of $R$ and also $L$ is essential in $R$. Now, $g(x) L=0$. Since $M$ is nonsingular then $g(x)=0$, and since $x$ is arbitrary $g=0$. Therefore $\Delta=0$ ([13, Lemma 2.3]). Hence the result follows from Theorem 2.16.

The following example shows that the converse of Corollary 2.16 does not hold. Moreover this example explains that endomorphism ring of a PIextending (and hence self-p-injective) module need not to be Abelian.

\section{Example 2.17.}

(i) Let $T=M_{2}(R)$ be the ring in [19, Example 4.77]. Note that $T_{T}$ is nonsingular self-p-injective which is not CS-module. Since $T$ is regular, so does $S=\operatorname{End}\left(T_{T}\right)$. However $T$ is not $\pi$-duo. Because if it were $\pi$-duo, then it would be a CS-module, a contradiction.

(ii) Let $V$ be a countably infinite dimensional vector space over a division ring $D$ and let $S=\operatorname{End}\left(V_{D}\right)$. Let $\left\{x_{1}, x_{2}, \ldots\right\}$ be a basis of $V$. It is clear that $V_{D}$ is PI-extending so it is self-p-injective. Since $\Delta=0, S$ is regular ring. However $S$ is not an Abelian ring. In fact, define $\sigma: V \rightarrow V$ by $\sigma\left(x_{i}\right)=x_{i+1}$ for all $i \geq 1$ and $\pi: V \rightarrow x_{i} D$ by $\pi\left(x_{i}\right)=x_{i}$ and $\pi\left(x_{j}\right)=0$ for $i \neq j$. Now $\sigma \pi\left(x_{i}\right)=\sigma\left(x_{i}\right)=x_{i+1}$ but $\pi \sigma\left(x_{i}\right)=\pi\left(x_{i+1}\right)=0$.

\section{Relatively p-injective Modules}

In this section we introduce the concept of a relative p-injective module and investigate some properties of these modules. Let us begin with the definition.

Definition 3.1. Let $M_{1}$ and $M_{2}$ be modules. The module $M_{2}$ is $M_{1}$-p-injective if every homomorphism $\alpha: N \rightarrow M_{2}$, where $N$ is a projection invariant submodule of $M_{1}$, can be extended to a homomorphism $\beta: M_{1} \rightarrow M_{2}$.

It is clear that relative p-injectivity is more general than relative injectivity. Next result provides equivalent conditions to be $P I$-extending in terms of relative p-injectivitiy.

Proposition 3.2. The following statements are equivalent for a module $M$.

(i) $M$ is PI-extending.

(ii) Every module is $M$-p-injective.

(iii) Every projection invariant submodule of $M$ is $M$-p-injective. 
Proof. $(i) \Rightarrow($ ii $)$ Let $X$ be a module and $N$ be a projection invariant submodule of $M$ with $\varphi: N \rightarrow X$ homomorphism. Since $M$ is $P I$-extending, there exists a direct summand $D$ of $M$ such that $N$ is essential in $D$. Then $M=D \oplus D^{\prime}$ for some $D^{\prime}$ submodule of $M$. Let $\pi$ be the projection map on $N$ in $D$. Define $\alpha: M \rightarrow X$ by $\alpha=\varphi \pi$. Clearly, $\alpha$ lifts to $\varphi \pi$ so $X$ is $M$-p-injective.

(ii) $\Rightarrow$ (iii) It is obvious.

$($ iii $) \Rightarrow(i)$ Let $N$ be a projection invariant submodule of $M$. By hypothesis, $N$ is $M$-p-injective so the identity map $\iota: N \rightarrow N$ can be extended to $\alpha: M \rightarrow$ $N$. It is easy to check that $M=N \oplus \operatorname{ker} \alpha$. Thus $M$ is $P I$-extending.

Next result, which generalizes $[4,7.5]$, concerns relative p-injective direct summands of a module.

Theorem 3.3. Let $M_{1}, M_{2}$ be modules and $M=M_{1} \oplus M_{2}$. Then $M_{2}$ is $M_{1}$-p-injective if and only if every submodule $N$ of $M$ such that $N \cap M_{2}=0$; and $\pi_{1}(N)$ is a projection invariant submodule of $M_{1}$, there exists a submodule $N^{\prime}$ of $M$ such that $N \leq N^{\prime}$ and $M=N^{\prime} \oplus M_{2}$.

Proof. Let $N \leq M$ such that $N \cap M_{2}=0$ and $\pi_{1}(N)$ is projection invariant submodule of $M_{1}$. Let $\pi_{1}: M \rightarrow M_{1}$ be the projection and consider the restriction of $\pi_{1}$ to $N$. Then $\left.\pi_{1}\right|_{N}$ is an isomorphism between $N$ to $\pi_{1}(N)$, since $N \cap M_{2}=0$. Consider the homomorphism $\alpha: \pi_{1}(N) \rightarrow M_{2}$ by $\alpha(x)=$ $\pi_{2}\left(\left.\pi_{1}\right|_{N}\right)^{-1}(x)$. Since $\pi_{1}(N)$ is projection invariant submodule of $M_{1}$ and $M_{2}$ is $M_{1}$-p-injective, the map $\alpha$ can be extended to a homomorphism $\beta$ : $M_{1} \rightarrow M_{2}$. Define $N^{\prime}=\left\{x+\beta(x) \mid x \in M_{1}\right\}$. Clearly, $N^{\prime}$ is a submodule of $M$ and $M=N^{\prime} \oplus M_{2}$. Let $n \in N$. $\beta \pi_{1}(x)=\alpha \pi_{1}(x)=\pi_{2}(x)$ and hence $x=\pi_{1}(x)+\pi_{2}(x)=\pi_{1}(x)+\beta \pi_{1}(x) \in N^{\prime}$. Then $N \leq N^{\prime}$.

Conversely, let $K$ be a projection invariant submodule of $M_{1}$ and $\alpha: K \rightarrow$ $M_{2}$ be a homomorphism. Define $N=\{x-\alpha(x) \mid x \in K\} . N$ is a submodule of $M$ and $N \cap M_{2}=0$. Moreover, it can be easily seen that $\pi_{1}(N)=K$. Hence $\pi_{1}(N)$ is projection invariant submodule of $M_{1}$. By hypothesis, there exists a submodule $N^{\prime}$ of $M$ such that $N \leq N^{\prime}$ and $M=N^{\prime} \oplus M_{2}$. Let $\pi: M \rightarrow M_{2}$ be projection with kernel $N^{\prime}$ and let $\beta: M_{1} \rightarrow M_{2}$ be the restriction of $\pi$ to $M_{1}$. Let $x \in K . \beta(x)=\pi(x)=\pi(x-\alpha(x)+\alpha(x))=\alpha(x)$. It follows that $M_{2}$ is $M_{1}$-p-injective.

Proposition 3.4. Let $\left\{M_{\lambda}\right\}_{\lambda \in \Lambda}$ be a family of $R$-modules. Then $X$ is $M_{\lambda}$-pinjective for all $\lambda \in \Lambda$ if and only if $X$ is $\underset{\lambda \in \Lambda}{\oplus} M_{\lambda}$-p-injective.

Proof. Let $M=\underset{\lambda \in \Lambda}{\oplus} M_{\lambda}$ and $\alpha: N \rightarrow A$ be homomorphism with a projection invariant submodule $N$ of $M$. Then $N=\underset{\lambda \in \Lambda}{\oplus}\left(N \cap M_{\lambda}\right)$ where $N \cap M_{\lambda}$ is 
projection invariant in $M_{\lambda}$. Consider the restriction of $\alpha$ on $N \cap M_{\lambda}$. Then there exists a homomorphism $\theta: M_{\lambda} \rightarrow X$ such that $\theta$ lifts to $\left.\alpha\right|_{N \cap M_{\lambda}}$. Define $\gamma: M \rightarrow X$ by $\gamma=\theta \pi_{\lambda}$ where $\pi_{\lambda}: M \rightarrow M_{\lambda}$ canonical projection. Then it can be easily seen that $\gamma$ lifts to $\alpha$, hence $X$ is $M$-p-injective.

Conversely, let $K_{\lambda}$ be projection invariant in $M_{\lambda}$ for any $\lambda \in \Lambda$ and $\alpha$ : $K_{\lambda} \rightarrow X$ be homomorphism. Then $K=\underset{\lambda \in \Lambda}{\oplus} K_{\lambda}$ is projection invariant in $M=\underset{\lambda \in \Lambda}{\oplus} M_{\lambda}$. Now, there exists a homomorphism $\theta: M \rightarrow X$ such that $\theta$ lifts to $\alpha \pi_{\lambda}$ where $\pi_{\lambda}$ is a projection map from $K$ onto $K_{\lambda}$. Define $\gamma: M_{\lambda} \rightarrow X$ by $\gamma=\theta \iota$ where $\iota$ is inclusion map. Then, it is clear that $\gamma$ lifts to $\alpha$. Therefore $X$ is $M_{\lambda}$-p-injective.

Our next two results give conditions for a module $X$ and its quotient being relative p-injective. To this end, we refer to $[2,16.8]$ for the corresponding relative injectivity results.

Theorem 3.5. Let $K$ be any projection invariant submodule of an $R$-module $M$. Then an $R$-module $X$ is $M$-p-injective if and only if

(i) $X$ is $K$-p-injective.

(ii) $X$ is $(M / K)$-p-injective.

(iii) any homomorphism $\varphi: K \rightarrow X$ can be lifted to a homomorphism $\theta: M \rightarrow X$.

Proof. Suppose that $X$ is $M$-p-injective. Then $(i)$ and (iii) clearly hold. Now suppose that $N / K$ is projection invariant in $M / K$ for $K \subseteq N \leq M$ and $\alpha$ : $N / K \rightarrow X$ is a homomorphism. Since $N / K$ is projection invariant in $M / K$, then $N$ is projection invariant in $M$. Let $\pi_{1}: M \rightarrow M / K$ and $\pi_{2}: N \rightarrow N / K$ be the canonical epimorphisms. Since $X$ is $M$-p-injective, the homomorphism $\alpha \pi_{2}: N \rightarrow X$ can be extended to the homomorphism $\gamma: M \rightarrow X$. Since $N \leq \operatorname{ker} \gamma$, there exists a homomorphism $\beta: M / K \rightarrow X$ such that $\beta \pi_{1}=\gamma$. Let $n \in N . \beta(n+K)=\beta\left(\pi_{1}(n)\right)=\gamma(n)=\alpha \pi_{2}(n)=\alpha(n+K)$. Hence $\beta$ lifts to $\alpha$ so $X$ is $(M / K)$-p-injective.

Conversely, suppose that $X$ satisfies $(i),(i i)$ and $(i i i)$. Let $N$ be projection invariant submodule of $M$ and $\varphi: N \rightarrow X$ be homomorphism. It is clear that $N \cap K$ is also projection invariant in $K$. Let $\varphi^{\prime}$ be the restriction of $\varphi$ to $N \cap K$. By $(i)$, there exists $\alpha: K \rightarrow X$ such that $\varphi^{\prime}$ can be lifted to $\alpha$. By (iii), there exists $\beta: M \rightarrow X$ such that $\beta$ lifts to $\alpha$. Thus

$$
\beta(k)=\alpha(k)=\varphi^{\prime}(k)=\varphi(k) \text { for all } k \in N \cap K .
$$

Let $\gamma=\varphi-\beta$. It is clear that $\gamma: N \rightarrow X$ and $\gamma(N \cap K)=0$. Define $\varphi^{\prime \prime}: N+K / K \rightarrow X$ by $\varphi^{\prime \prime}(n+K)=\gamma(n+K)$ for all $n \in N$. $\varphi^{\prime \prime}$ is well defined, since $\gamma(N \cap K)=0$. Note that $N+K$ and $N+K / K$ are both 
projection invariant in $M$. Thus by ( $i i)$, there exists $\theta^{\prime}: M / K \rightarrow X$ such that $\theta^{\prime}$ lifts to $\varphi^{\prime \prime}$. Define $\theta^{\prime \prime}: M \rightarrow X$ by $\theta^{\prime \prime}(m)=\theta^{\prime}(m+K)$ for all $m \in M$. Let $\theta=\beta+\theta^{\prime \prime}$ where $\theta: M \rightarrow X$. Let $n \in N$. Then

$$
\theta(n)=\beta(n)+\theta^{\prime \prime}(n)=\varphi(n)-\varphi^{\prime \prime}(n+K)+\theta^{\prime}(n+K)=\varphi(n) .
$$

Thus $\theta$ lifts $\varphi$ so $X$ is $M$-p-injective.

Proposition 3.6. Let $K \subseteq N$ be submodules of an $R$-module $M$. Then the following statements are equivalent.

(i) $N / K$ is $M$-p-injective.

(ii) For all $K \leq N \leq M$ with projection invariant submodule $N / K$ of $M / K, N / K$ is a direct summand of $M / K$.

Proof. $(i) \Rightarrow$ (ii) $N / K$ is $M / K$-p-injective by Theorem 3.5 . Thus the identity map $\iota: N / K \rightarrow N / K$ can be lifted to a homorphism $\theta: M / K \rightarrow N / K$. It is easy to check that $M / K=\operatorname{ker} \theta \oplus(N / K)$.

$($ ii $) \Rightarrow(i)$ Let $X$ be a projection invariant submodule of $M$ and $\varphi: X \rightarrow$ $N / K$ be a homomorphism with $K=\operatorname{ker} \varphi$. Since $X / K$ is projection invariant in $M / K$ and $K=\operatorname{ker} \varphi \leq X \leq A$, then $X / K$ is a direct summand of $M / K$ by (ii). Thus there exists $L \leq M$ such that $K \subseteq L, M / K=X / K \oplus L / K$. Define $\theta: M \rightarrow N / K$ by $\theta(x+l)=\varphi(x)$ where $x \in X$ and $l \in L$. Note that if $x \in X$, $l \in L$ and $x+l=0$, then $x=-l \in X \cap L=K$. Thus $\theta$ is well defined. Clearly $\theta$ is an $R$-homomorphism and $\theta$ lifts $\varphi$. Thus $N / K$ is $M$-p-injective.

\section{Examples}

We provide examples which show that self-p-injective and tight are different notions. Recall that 2-by-2 upper triangular matrix ring over a field is a right CS ring by [18, Theorem 3.4]. To this end, the following example corrects [6, Example 2.11].

Example 4.1. Let $S=\mathbb{Z}[x]$ and let $R$ be the 2-by-2 full matrix ring over $S$. Then $R$ is not right CS by [4, Lemma 12.8]. By [10, Corollary 11.18 and Corollary 11.19], $R$ is a semiprime right Goldie ring. Then $R$ is $R$-tight by [7].

There is a self-p-injective module which is not tight (see, [1, Example 3.1]). However the following example (see, [6, Example 2.13]) is $R$-tight but it is not self-p-injective.

Example 4.2. Let $R=\{(m, n) \mid m \equiv n(\bmod 2)\} \subseteq \mathbb{Z} \times \mathbb{Z}$. Then $R_{R}$ is tight by [6, Example 2.13]. Since $R$ is indecomposable, Lemma 2.1 yields that $R$ is not self-p-injective. 
Acknowledgment. The authors would like to express their appreciation to the referee for his/her careful reading to the paper and useful suggestions.

\section{References}

[1] Al-Huzali, A., Jain, S. K., Lopez-Permouth, S. R., On the weak relativeinjectivity of rings and modules, Noncommutative ring theory, Lecture Notes in Math., 1448, Springer, Berlin, 1990.

[2] Anderson, F. W., Fuller, K. R., Rings and Categories of Modules, New York, Springer-Verlag, 1974.

[3] Birkenmeier, G. F., Tercan, A., Yücel, C. C., The extending condition relative to sets of submodules, Comm. Algebra 42 (2014) 764-778.

[4] Dung, N. V., Huynh, D. V., Smith, P. F., Wisbauer, R., Extending Modules, Pitman, London, 1994.

[5] Fuchs, L., Infinite Abelian Groups I, New York, Academic Press, 1970.

[6] Guil Asensio, P. A., Sahinkaya, S., Srivastava, A. K., New characterizations of pseudo-Frobenius rings and a generalization of the FGF conjecture, Isr. J. Math., 214 (2016), 121-148.

[7] Jain, S. K., Lopez-Permouth, S. R., A survey on the theory of weakly injective modules, in Computational Algebra, Lecture Notes Pure Appl. Math. 151, Marcel Dekker, New York, (1993) 205-232.

[8] Kaplansky, I. Infinite Abelian Groups, University of Michigan Press, 1969.

[9] Kara, Y., Tercan, A., Modules whose certain submodules are essentially embedded in direct summands, Rocky Mt. J. Math., 46(2) (2016) 519-532.

[10] Lam, T. Y., Lectures on Modules and Rings, Springer-Verlag, 1999.

[11] Mohamed, S. H., Müller, B. J., Continuous and Discrete Modules, London Math. Soc. 147, 1990.

[12] Murthy, M. P., Zero cycles and projective modules, Ann. Math., (1994) 405-434.

[13] Sandomierski, F.L., Non-singular rings, Proc. Amer. Math. Soc. 19 (1968) 225-230. 
[14] Santa-Clara, C., Smith, P. F., Modules which are self-injective relative to closed submodules, Algebra and Its Applications, Contemp. Math. Athens, Ohio, 259 (1999) 487499.

[15] Smith, P.F., Tercan, A., Generalizations of CS-modules, Comm. Algebra 21 (1993) 1809-1847.

[16] Smith, P.F., Tercan, A., Continuous and quasi-continuous modules, Houston J. Math., 18(3) (1992) 339-348.

[17] Smith, P.F., Tercan, A., Direct summands of modules which satisfy $\mathrm{C}_{11}$, Algebra Colloq., 11 (2004) 231-237.

[18] Tercan, A., On certain CS rings, Comm. Algebra 23(2) (1995) 405-419.

[19] Tercan, A., Yücel, C. C., Module Theory, Extending Modules and Generalizations, Basel, Birkhäuser, 2016.

Yeliz KARA

Hacettepe University,

Department of Mathematics,

Beytepe Campus, Ankara 06532, Turkey

Email:yelizkara@hacettepe.edu.tr

Adnan TERCAN

Hacettepe University,

Department of Mathematics,

Beytepe Campus, Ankara 06532, Turkey

Email:tercan@hacettepe.edu.tr 